MITSUBISHI ELECTRIC RESEARCH LABORATORIES

http://www.merl.com

\title{
Laser Frequency Drift Compensation with Han-Kobayashi Coding in Superchannel Nonlinear Optical Communications
}

\author{
Koike-Akino, T.; Millar, D.S.; Kojima, K.; Parsons, K.
}

TR2015-110 September 2015

\begin{abstract}
We investigate the impact of laser frequency drift, which can cause inter-channel interference (ICI) in superchannel communications. We show that Han-Kobayashi coding significantly improves robustness against the drift, achieving 2-times higher achievable rate than conventional coding.
\end{abstract}

2015 European Conference on Optical Communication (ECOC)

This work may not be copied or reproduced in whole or in part for any commercial purpose. Permission to copy in whole or in part without payment of fee is granted for nonprofit educational and research purposes provided that all such whole or partial copies include the following: a notice that such copying is by permission of Mitsubishi Electric Research Laboratories, Inc.; an acknowledgment of the authors and individual contributions to the work; and all applicable portions of the copyright notice. Copying, reproduction, or republishing for any other purpose shall require a license with payment of fee to Mitsubishi Electric Research Laboratories, Inc. All rights reserved. 



\title{
Laser Frequency Drift Compensation with Han-Kobayashi Coding in Superchannel Nonlinear Optical Communications
}

\author{
Toshiaki Koike-Akino, David S. Millar, Keisuke Kojima, Kieran Parsons
}

Mitsubishi Electric Research Labs., 201 Broadway, Cambridge, MA 02139, USA, koike@merl.com

\begin{abstract}
We investigate the impact of laser frequency drift, which can cause inter-channel interference (ICl) in superchannel communications. We show that Han-Kobayashi coding significantly improves robustness against the drift, achieving 2-times higher achievable rate than conventional coding.
\end{abstract}

\section{Introduction}

The demand of high-speed data rates in optical communications has brought advanced technologies including superchannel transmission ${ }^{1-6}$, where parallel transmitters send independent data using different wavelengths to increase total throughput. The spectral efficiency increases as the channel spacing decreases. However, interchannel interference $(\mathrm{ICl})$ can be a major limiting factor to realize dense channel allocation.

In order to handle ICI in superchannel transmissions, we have proposed to use Han-Kobayashi $(\mathrm{HK})$ coding $^{6}$, which showed significant gain in spectral efficiency in the presence of strong $\mathrm{ICl}$ for super-dense sub-Nyquist channel spacings. It was expected that the HK coding will enable increased robustness to other practical hardware imperfections such as laser frequency drift and mistuning, which can cause probabilistic ICI even for quasi-Nyquist channel spacings.

This paper studies the gain of the HK coding in the presence of laser frequency drift. We show that the spectral efficiency can be improved by the HK coding, especially when a large deviation of laser frequency is present. This may reduce requirements for laser frequency stabilization, leading to lower complexity and power consumption.

\section{Superchannels with laser frequency drift}

We consider a superchannel transmission system with $N_{\text {ch }}=3$ subchannels. Fig. 1 shows an example of power spectrum of superchannel signals for the case when the channel spacing normalized by baud rate $B$ is $\delta f=1.01$ so that no $\mathrm{ICl}$ is present with a root-raised-cosine (RRC) filter of rolloff factor 0.01. Although such a quasi-Nyquist system achieves the highest spectral efficiency, the system can be readily suffered from some hardware imperfections such as laser frequency drift. It is shown in Fig. 1 that undesired $\mathrm{ICl}$ across adjacent subchannels can occur if the transmitter ( $\mathrm{Tx}$ ) laser frequency is deviated from the nominal case. For

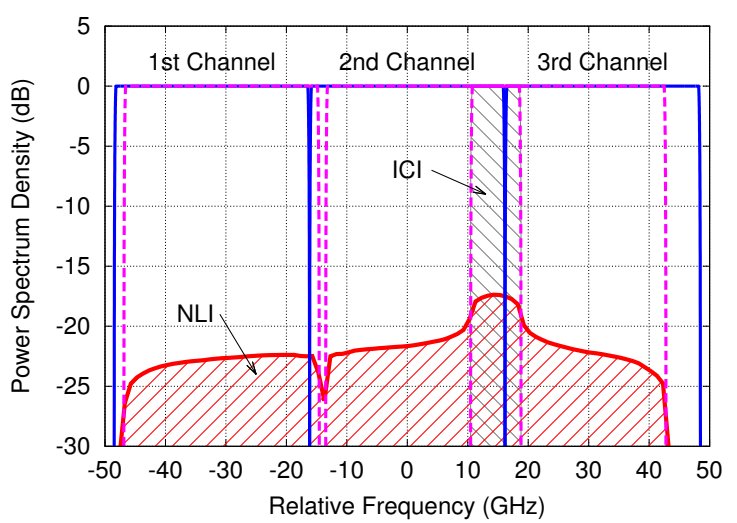

Fig. 1: Spectrum example of superchannel transmission in presence of laser frequency drift $\left(\delta f=1.01, \sigma_{f}=0.1\right)$. Blue solid line: nominal Tx spectrum, pink dashed line: deviated

Tx spectrum due to frequency drift, black-shaded area: possible $\mathrm{ICI}$, red-shaded area: NLI computed by GN model ${ }^{7}$.

simplicity, we assume that the laser frequency is randomly drifted by following the Gaussian distribution with a standard deviation of $\sigma_{f} B$.

As shown in Fig. 1, nonlinear interference (NLI) caused by Kerr fiber nonlinearity also deviates according to Tx spectrum. Here, the NLI spectrum is calculated by the Gaussian noise (GN) model ${ }^{7}$. Since the NLI can be non-identical for different subchannels, rate adaptation ${ }^{5}$ and/or power control $^{4}$ have been studied to improve performance. Without such rate/power controls, the achievable rate of the conventional coding scheme can be constrained by the worst subchannel as follows:

$$
R_{\text {conv }}=N_{\text {ch }} \min _{k}\left[C\left(\frac{\rho_{k} \beta_{k, k}}{1+\rho_{k} \sum_{i \neq k} \beta_{i, k}}\right)\right],
$$

where $C(x)=\log _{2}(1+x), \rho_{k}$ is the signal-to-noise ratio at the $k$-th subchannel, and $\beta_{i, k}$ corresponds to a power fraction from the $i$-th Tx subchannel to the $k$-th receiver ( $\mathrm{Rx}$ ) subchannel, defined as

$$
\beta_{i, k}=\frac{\int\left|G_{\mathrm{rrc}}\left(f-f_{i}^{\mathrm{tx}}\right) H_{\mathrm{cd}}(f) G_{\mathrm{rrc}}\left(f-f_{k}^{\mathrm{rx}}\right)\right|^{2} \mathrm{~d} f}{\int\left|G_{\mathrm{rrc}}\left(f-f_{k}^{\text {nom }}\right) H_{\mathrm{cd}}(f) G_{\mathrm{rrc}}\left(f-f_{k}^{\text {nom }}\right)\right|^{2} \mathrm{~d} f},
$$

where $G_{\mathrm{rrc}}(f)$ and $H_{\mathrm{cd}}(f)$ denote transfer func- 
tions of the RRC filter and chromatic dispersion, respectively. Here, $f_{k}^{\mathrm{tx}}, f_{k}^{\mathrm{rx}}$, and $f_{k}^{\text {nom }}$ are carrier frequencies for the Tx laser, Rx laser, and nominal case, respectively, at the $k$-th subchannel. Because frequency drifts occur at both $\mathrm{Tx}$ and $\mathrm{Rx}$ lasers (i.e., $f_{k}^{\mathrm{tx}} \neq f_{k}^{\mathrm{rx}}$ with high probability), the desired signal power can be also deviated as $\beta_{k, k}<1$. The ICl from the $i$-th subchannel to the $k$-th subchannel can appear when the $\mathrm{Tx}$ and $\mathrm{Rx}$ filters overlap each other as $\beta_{i, k}>0$ for $i \neq k$.

\section{Han-Kobayashi (HK) superchannel coding}

To deal with $\mathrm{ICl}$ caused by the laser frequency drift, we consider superchannel optical transmission systems employing the HK coding scheme ${ }^{6}$, as shown in Fig. 2. The HK scheme splits data at each Tx subchannel into two portions; one is private data $u_{n}$ for only the intended $\mathrm{Rx}$, and the other data $w_{n}$ is public for all Rx's. These two data are superimposed with a certain power splitting ratio $\lambda_{n}$. At each $\mathrm{Rx}$ subchannel, all public data are jointly decoded, and intended private data is decoded after ICl cancellation. Unintended public data (i.e., $w_{i}$ for $i \neq n$ at the $n$-th Rx subchannel) are discarded in the end.

By controlling the power splitting ratio $\lambda_{n}$, the HK scheme can achieve joint decoding gain for public data while mitigating $\mathrm{ICl}$ for private data. The achievable sum rate is expressed as follows:

$$
\begin{aligned}
& R_{\mathrm{HK}}= \sum_{k} C\left(\frac{\rho_{k} \lambda_{k} \beta_{k, k}}{1+\rho_{k} \sum_{i \neq k} \lambda_{i} \beta_{i, k}}\right) \\
&+\min _{k}\left[C\left(\rho_{k}^{\prime} \sum_{i} \bar{\lambda}_{i} \beta_{i, k}\right), \sum_{j} C\left(\rho_{k}^{\prime} \bar{\lambda}_{j} \beta_{j, k}\right),\right. \\
&\left.\min _{j}\left\{C\left(\rho_{k}^{\prime} \bar{\lambda}_{j} \beta_{j, k}\right)+C\left(\rho_{k}^{\prime} \sum_{i \neq j} \bar{\lambda}_{i} \beta_{i, k}\right)\right\}\right],
\end{aligned}
$$

where $\bar{\lambda}_{k}=1-\lambda_{k}$ and $\rho_{k}^{\prime}=\rho_{k} /\left(1+\rho_{k} \sum_{i} \lambda_{i} \beta_{i, k}\right)$. The first term of $R_{\mathrm{HK}}$ comes from the private data $u_{n}$ after $\mathrm{ICl}$ cancellation, and the reminder corresponds to joint decoding of public data $w_{n}$.

\section{System parameters}

We assume $B=32$ Gbaud per channel and RRC filter with a rolloff factor of $\alpha=0.01$. The normalized channel spacing $\delta f$ is chosen from $0.5 \leq$ $\delta f \leq 1.2$. We use the $\mathrm{GN}$ model $^{7}$ to calculate NLI power spectrum after $N_{\mathrm{s}}=15$ spans of standard single-mode fiber (SSMF), whose span length is $80 \mathrm{~km}$, dispersion parameter is $16.5 \mathrm{ps} / \mathrm{nm} / \mathrm{km}$, fiber loss is $0.2 \mathrm{~dB} / \mathrm{km}$, and nonlinear coefficient is $1.3 / \mathrm{W} / \mathrm{km}$. Amplified spontaneous emission noise is calculated, assuming that Erbium-doped fiber

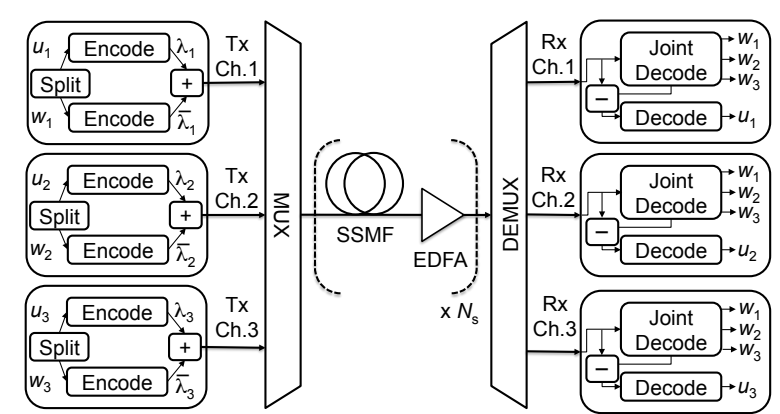

Fig. 2: Superchannel optical communications with Han-Kobayashi coding for ICl management.

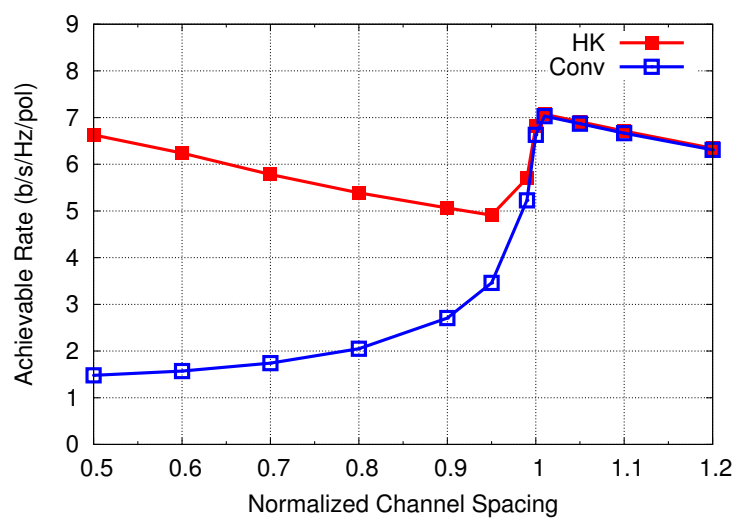

Fig. 3: Nominal spectral efficiency as a function of normalized channel spacing $\delta f$ with no laser frequency drift.

amplifier (EDFA) with a noise figure of $4 \mathrm{~dB}$ compensates for every span loss. The theoretical analysis is carried out by calculating NLI according to randomly deviated laser frequencies. The achievable sum rate is normalized by a nominal superchannel bandwidth of $B\left(1+\alpha+\delta f\left(N_{\mathrm{ch}}-1\right)\right)$.

\section{Performance results}

Fig. 3 shows the achievable rate as a function of channel spacing $\delta f$, for the nominal case without frequency drifts. It is shown that the spectral efficiency is maximized at a quasi-Nyquist spacing of $\delta f=1.01$. Although the HK coding can compensate for $\mathrm{ICl}$ at sub-Nyquist spacings of $\delta f<1$, the achievable rate cannot be higher than the zero- $\mathrm{ICI}$ cases. However, the quasi-Nyquist spacing may be susceptible to laser frequency drifts. In fact, the laser frequency can drift by a few $\mathrm{GHz}$ due to aging and other effects if no frequency stabilizer other than temperature controller is used.

Fig. 4 shows the outage probability of achievable rates at a channel spacing of $\delta f=1.01$ in the presence of laser frequency drifts with a standard deviation of $\sigma_{f}=10,1,0.1 \%$. We can see that the achievable rate is significantly degraded as the standard deviation $\sigma_{f}$ increases. Fig. 5 shows the achievable rate as a function of launch power for $\delta f=1.01$ and $\sigma_{f}=1 \%$. For achieving an outage probability below $1 \%$, the HK coding is advantageous to compensate for the loss caused by laser 


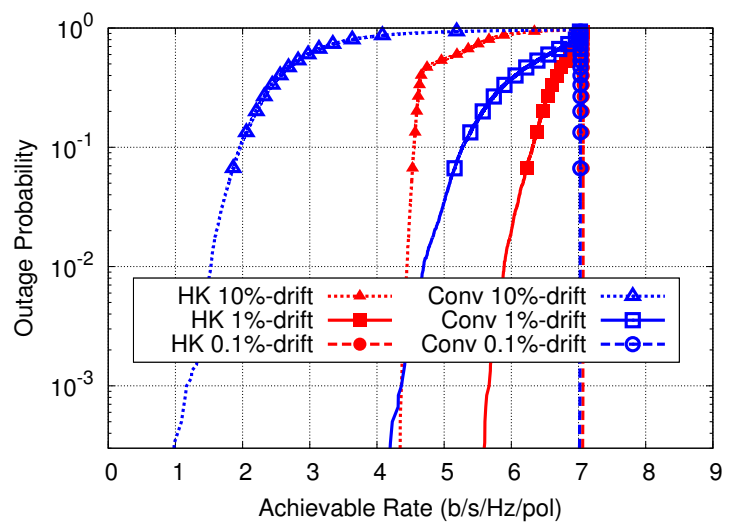

Fig. 4: Outage probability of achievable rate in presence of laser frequency drift $\left(\delta f=1.01, \sigma_{f}=0.10,0.01,0.001\right)$.

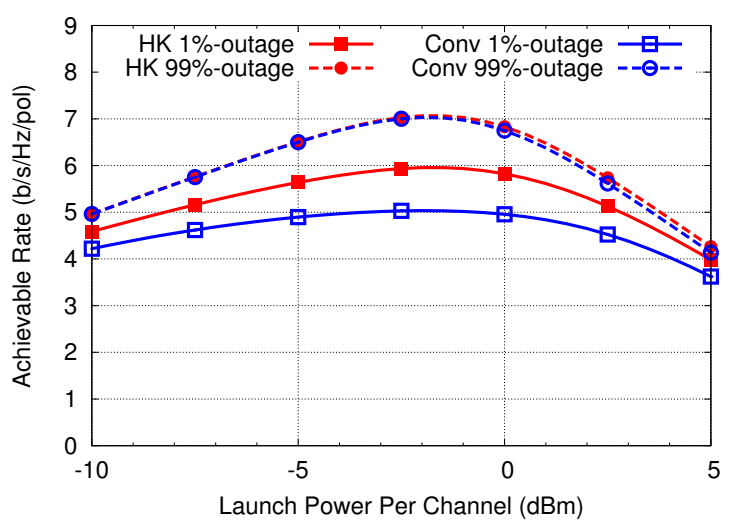

Fig. 5: Achievable rate as a function of launch power in presence of laser frequency $\operatorname{drift}\left(\delta f=1.01, \sigma_{f}=0.01\right)$.

frequency drifts even for quasi-Nyquist spacings. Note that practical systems require more stringent outage probability below $10^{-7}$. It is expected from Fig. 4 that the gain of the HK coding can be higher for lower outage probabilities.

Figs. 6 and 7 show the achievable rates for $1 \%$ outage as a function of laser frequency deviation $\sigma_{f}$ and normalized channel spacing $\delta f$, respectively. The conventional scheme at a quasiNyquist spacing of $\delta f>1$ showed considerable rate degradation for a frequency deviation above $\sigma_{f}>0.3 \%$. Note that the HK coding at a subNyquist spacing of $\delta f=0.7$ can outperform the low-density spacing cases at $\delta f>1$ in the presence of a large laser frequency drift of $\sigma_{f}=10 \%$.

\section{Conclusions}

We analyzed the impact of laser frequency drift on the achievable rate for superchannel optical communications. It was shown that the HK coding scheme can provide improved robustness against $\mathrm{ICl}$ caused by the laser frequency drift, achieving 2-times higher rate than conventional scheme. In addition, sub-Nyquist $70 \%$ spacing can maintain $5 \mathrm{~b} / \mathrm{s} / \mathrm{Hz} / \mathrm{pol}$ spectral efficiency, and outperform quasi-Nyquist systems at a $10 \%$ standard deviation of laser frequency. Such high robustness

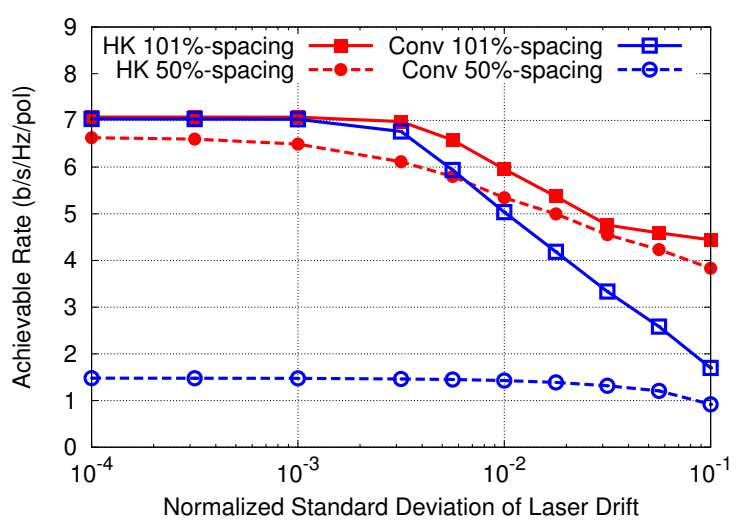

Fig. 6: Achievable rate for $1 \%$ outage as a function of laser frequency deviation $\sigma_{f}(\delta f=0.50,1.01)$.

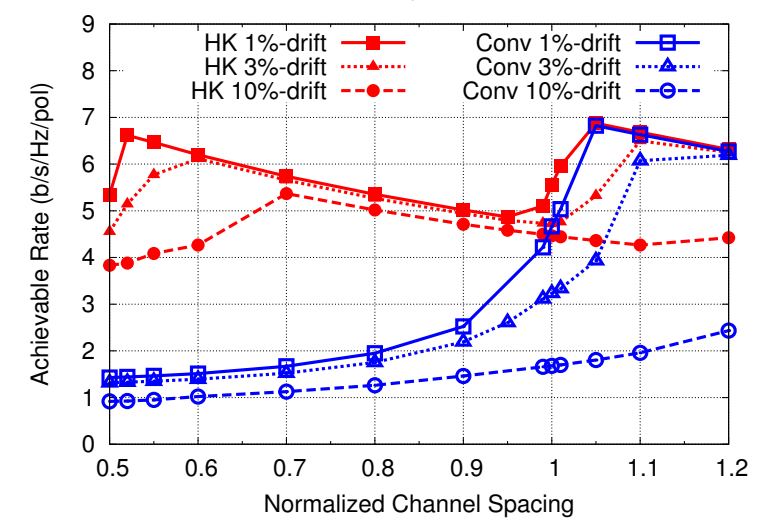

Fig. 7: Achievable rate for $1 \%$ outage as a function of normalized channel spacing $\delta f\left(\sigma_{f}=0.1,0.03,0.01\right)$.

to the drift may reduce the complexity and power consumption for laser frequency stabilization.

\section{References}

[1] J. Li et al., "1.94 Tb/s $(11 \times 176 \mathrm{~Gb} / \mathrm{s})$ DP-16QAM superchannel transmission over $640 \mathrm{~km}$ EDFA-only SSMF and two 280GHz WSSs," ECOC, Th.2.C.1 (2012).

[2] J.H. Ke, Y. Gao, J.C. Cartledge, "400 Gbit/s single-carrier and $1 \mathrm{Tbit} / \mathrm{s}$ three-carrier superchannel signals using dual polarization 16-QAM with look-up table correction and optical pulse shaping," Optics Express, 2271 (2014).

[3] X. Liu et al., "1.5-Tb/s guard-banded superchannel transmission over $56 \times 100-\mathrm{km}(5600-\mathrm{km})$ ULAF using 30 Gbaud pilot-free OFDM-16QAM signals with $5.75-\mathrm{b} / \mathrm{s} / \mathrm{Hz}$ net spectral efficiency," ECOC, Th.3.C.5 (2012).

[4] O. Vassilieva et al., "Systematic analysis of intrasuperchannel nonlinear crosstalk in flexible grid networks," ECOC, Mo.4.3.6 (2014).

[5] K. Kojima et al., "Maximizing transmission capacity of superchannels using rate-adaptive FEC," ECOC, P.3.23 (2014).

[6] T. Koike-Akino et al., "Han-Kobayashi and dirtypaper coding for superchannel optical communications," IEEE/OSA JLT, 337 (2015).

[7] P. Poggiolini, "The GN model of non-linear propagation in uncompensated coherent optical systems," IEEE/OSA JLT, 30 24, 3857-3879 (2012). 